\title{
INTERCEPTAÇÃO DE RADIAÇÃO SOLAR E DISTRIBUIÇÃO ESPACIAL DE ÁREA FOLIAR EM FLORESTA DE TERRA FIRME DA AMAZÔNIA CENTRAL, BRASIL
}

\author{
Arí de O. MARQUES FILHO', Ricardo G. DALLAROSA ${ }^{1}$
}

\begin{abstract}
RESUMO - A ocorrência de radiação solar em meio florestal e a sua relação com a distribuição espacial dos elementos vegetais são investigadas neste trabalho. Técnicas experimentais de amostragem de radiação no interior de coberturas vegetais de grande porte são desenvolvidas e testadas, tendo como base dispositivos de suporte móveis constituidos por reticulados modulares suspensos na parte superior das grandes árvores, que permitem a disposição dos sensores de radiação em diferentes niveis de uma mesma vertical e a replicação das medidas em diferentes verticais no interior da cobertura. As medidas de radiação solar foram realizadas no sítio experimental da Reserva Florestal Ducke (02 $56^{\prime}$ S; 59 $57^{\prime}$ W), Manaus - Brasil, no período de 29 de outubro a 11 de dezembro de 1998. A inversão de modelo físico de radiação permitiu o estabelecimento da função de densidade de área foliar para a vegetação do local. Os valores obtidos para o indice de área foliar (IAF) nas três verticais em condições de radiação difusa foram 6,6 , 6,3 e 6,2 .
\end{abstract}

Palavras-chave: índice de área foliar, radiação solar, floresta, Amazônia.

Solar Radiation Interception and Spatial Leaf Area Distribution in 'Terra Firme' Forest of the Central Amazonia, Brazil

ABSTRACT - The occurrence of solar radiation in forest medium and its relationship with the spatial distribution of the canopy elements are investigated in this work. Experimental sampling techniques of the radiation measurements inside tall vegetation covers are developed and tested, having as base mobile support apparatus, made of modular reticulates suspended on the top of the large trees, which allows the placement of the radiation sensors at different heights along a vertical profile within the canopy and the replication of the vertical probing at different sites. Solar radiation measurements were made at the experimental site of Reserva Ducke $\left(02^{\circ} 56^{\prime}\right.$ S; 59 57' W), Manaus - Brazil, in the period 29 Oct. to 11 Dec. 1998. The inversion of the solar radiation physical model allowed the estimation of the leaf area density for the local vegetation. The calculated values for the leaf area index (LAI) at the three verticals with dominant diffuse radiation conditions were $6.6,6.3$ e 6.2 .

Key-words: leaf area index, solar radiation, forest, Amazonia.

\section{INTRODUÇÃO}

Os fenômenos físicos que ocorrem na passagem da radiação solar em um meio vegetal são bem conhecidos e estabelecidos de forma adequada em termos teóricos, compreendendo no caso mais completo e geral, descrições consistentes da interceptação, da absorção e do espalhamento da radiação incidente pelos elementos vegetais constituintes da cobertura, especialmente as folhas com seu desenvolvimento superficial bem superior aos dos galhos e troncos das árvores. Esses conceitos teóricos sobre a radiação solar em meio vegetal são reunidos em modelos físicos explicitados de forma analítica (Nilson, 1971; Ross, 1981; Myneni et al., 1989) e apropriados à inversão

\footnotetext{
'Instituto Nacional de Pesquisas da Amazônia - INPA - Caixa Postal 478 - Manaus - AM
} 


\title{
INTERCEPTAÇÃO DE RADIAÇÃO SOLAR E DISTRIBUIÇÃO ESPACIAL DE ÁREA FOLIAR EM FLORESTA DE TERRA FIRME DA AMAZÔNIA CENTRAL, BRASIL
}

\author{
Arí de O. MARQUES FILHO', Ricardo G. DALLAROSA ${ }^{1}$
}

\begin{abstract}
RESUMO - A ocorrência de radiação solar em meio florestal e a sua relação com a distribuição espacial dos elementos vegetais são investigadas neste trabalho. Técnicas experimentais de amostragem de radiação no interior de coberturas vegetais de grande porte são desenvolvidas e testadas, tendo como base dispositivos de suporte móveis constituidos por reticulados modulares suspensos na parte superior das grandes árvores, que permitem a disposição dos sensores de radiação em diferentes niveis de uma mesma vertical e a replicação das medidas em diferentes verticais no interior da cobertura. As medidas de radiação solar foram realizadas no sítio experimental da Reserva Florestal Ducke (02 $56^{\prime}$ S; 59 $57^{\prime}$ W), Manaus - Brasil, no período de 29 de outubro a 11 de dezembro de 1998. A inversão de modelo físico de radiação permitiu o estabelecimento da função de densidade de área foliar para a vegetação do local. Os valores obtidos para o indice de área foliar (IAF) nas três verticais em condições de radiação difusa foram 6,6 , 6,3 e 6,2 .
\end{abstract}

Palavras-chave: índice de área foliar, radiação solar, floresta, Amazônia.

Solar Radiation Interception and Spatial Leaf Area Distribution in 'Terra Firme' Forest of the Central Amazonia, Brazil

ABSTRACT - The occurrence of solar radiation in forest medium and its relationship with the spatial distribution of the canopy elements are investigated in this work. Experimental sampling techniques of the radiation measurements inside tall vegetation covers are developed and tested, having as base mobile support apparatus, made of modular reticulates suspended on the top of the large trees, which allows the placement of the radiation sensors at different heights along a vertical profile within the canopy and the replication of the vertical probing at different sites. Solar radiation measurements were made at the experimental site of Reserva Ducke $\left(02^{\circ} 56^{\prime}\right.$ S; 59 57' W), Manaus - Brazil, in the period 29 Oct. to 11 Dec. 1998. The inversion of the solar radiation physical model allowed the estimation of the leaf area density for the local vegetation. The calculated values for the leaf area index (LAI) at the three verticals with dominant diffuse radiation conditions were $6.6,6.3$ e 6.2 .

Key-words: leaf area index, solar radiation, forest, Amazonia.

\section{INTRODUÇÃO}

Os fenômenos físicos que ocorrem na passagem da radiação solar em um meio vegetal são bem conhecidos e estabelecidos de forma adequada em termos teóricos, compreendendo no caso mais completo e geral, descrições consistentes da interceptação, da absorção e do espalhamento da radiação incidente pelos elementos vegetais constituintes da cobertura, especialmente as folhas com seu desenvolvimento superficial bem superior aos dos galhos e troncos das árvores. Esses conceitos teóricos sobre a radiação solar em meio vegetal são reunidos em modelos físicos explicitados de forma analítica (Nilson, 1971; Ross, 1981; Myneni et al., 1989) e apropriados à inversão

\footnotetext{
'Instituto Nacional de Pesquisas da Amazônia - INPA - Caixa Postal 478 - Manaus - AM
} 
matemática para a derivação de características principais da vegetação (índice de área foliar, IAF; distribuição vertical de densidade de área foliar) (Welles \& Norman, 1991; Andrieu \& Baret, 1993). A compreensão e o estabelecimento do regime de radiação no interior da cobertura também contribuem primariamente para a definição da distribuição de energia no interior dessa cobertura, o que constitui um dos fatores fundamentais nos estudos sobre os intercâmbios energéticos e de massa entre o corpo vegetal e a atmosfera.

A crescente necessidade de uma completa representação física das complexas caracteristicas ambientais e biológicas internamente ao dossel motivou uma série de investigações sobre o comportamento das diversas variáveis no perfil desde a superfície do solo até o topo da vegetação. Com isso, as medidas micrometeorológicas realizadas ao longo desse perfil ganharam destaque, impulsionadas também pelo desenvolvimento de sensores de grande sensibilidade e precisão e de sistemas inteligentes de coleta, processamento armazenamento desses dados.

Estudos dessa natureza têm sido realizados em coberturas vegetais de médio e grande porte na Amazônia, constituindo-se de determinações do índice de área foliar e da distribuição vertical da função $a(z)$ (densidade de área foliar) por métodos indiretos baseados na penetração de radiação solar no meio vegetal (Honzák et al., 1996; Wandelli \& Marques Filho,
1999) que se posicionam como importantes alternativas aos métodos destrutivos e trabalhosos de medida direta de toda área foliar existente numa área amostral escolhida (McWilliam et al., 1993).

Honzák et al. desenvolveram uma análise sobre métodos de determinação do IAF e compararam estimativas desse indice para diferentes coberturas vegetais da Amazônia, obtidas pela combinação de modelos particulares de atenuação da radiação solar em meio vegetal com medidas de radiação fotossinteticamente ativa, contagem de manchas de radiação junto ao solo e fotografias hemisféricas do interior da vegetação.

As variações temporais da radiação solar e a distribuição vertical de área foliar em florestas foram medidas e estudadas com o suporte de torre metálica que sobressai acima das copas das árvores e sobre a qual foram dispostos os sensores de radiação em diferentes níveis no interior da cobertura (Marques Filho, 1997). As dificuldades principais em tais tipos de experimentos estão sempre associadas à proximidade inevitável entre os sensores e os elementos estruturais da torre (afastamentos normais de 5 a 6 $m)$. No domínio da interação radiação solar - vegetação, a presença da torre com seus patamares regularmente distribuídos na vertical incorpora superficies adicionais (estranhas à vegetação), que interceptam radiação e cujos os efeitos sobre as medidas não podem ser separados facilmente da atenuação natural da radiação 
imposta pela vegetação.

Inserido nessa temática, o presente estudo tem como objetivos primários: (i) analisar o comportamento da radiação solar no interior de floresta, enfocando a sua variabilidade espaço-temporal e os fatores que determinam a sua atenuação na direção vertical; (ii) inverter modelo físico do regime de radiação solar em meio vegetal para a derivação da função $\mathrm{a}(\mathrm{z})$ de densidade de área foliar média para a cobertura; (iii) apresentar dispositivo experimental de medidas de radiação solar no interior de coberturas vegetais de grande porte, que elimina a influência sobre as medidas de estruturas robustas similares a torres micrometeorológicas e que favorece abordagens da variabilidade espacial de grandezas físicas no interior da vegetação pelo uso de múltiplas verticais num mesmo sítio experimental.

\section{MATERIAL E MÉTODOS}

\section{A) Área de estudo}

A área de estudo localiza-se na Reserva Florestal Adolpho Ducke, um sitio de floresta tropical com superficie total de $100 \mathrm{~km}^{2}$, distante cerca de 25 $\mathrm{km}$ ao norte de Manaus, $\left(02^{\circ} 56^{\prime} \mathrm{S}\right.$; $\left.59^{\circ} 57^{\prime} \mathrm{W}\right)$. A vegetação apresenta estrutura de floresta primária não perturbada, com altura média de $35 \mathrm{~m}$ e cujos espécimes mais emergentes chegam a atingir $40 \mathrm{~m}$. O terreno é levemente ondulado com uma altitude média em torno dos $80 \mathrm{~m}$ (datum existente na área indica $84 \mathrm{~m}$ ) e com vales de umas poucas dezenas de metros ocorrendo em intervalos de cerca de $300 \mathrm{~m}$. As normais extraídas de uma série de dados climatológicos (1965/1994) apontam duas estações anuais, sendo uma seca (junho a novembro) com normal mínima total mensal de $93 \mathrm{~mm}$, em julho, e uma chuvosa (dezembro a maio) com normal máxima total mensal de $299 \mathrm{~mm}$, em fevereiro. As temperaturas com média diária anual de $26,7^{\circ} \mathrm{C}$ apresentam uma amplitude média de $9,6^{\circ} \mathrm{C}$ (maior amplitude térmica em setembro, com $11,1^{\circ} \mathrm{C}$ e menor em abril, com $8,2^{\circ} \mathrm{C}$ ), sendo os meses de setembro e outubro os mais quentes (Tmáx média de $33^{\circ} \mathrm{C}$ ) e, o mais frio, julho (Tmín média de $21,3^{\circ} \mathrm{C}$ ). A umidade relativa apresenta indices médios mensais acima dos $80 \%$, sendo setembro a menor média ( $84 \%)$ e maio a maior $(91 \%)$. Os ventos próximos à superfície são bastante fracos e descrevem um comportamento característico zonal da região (direção $\mathrm{E} / \mathrm{NE}$ ), chegando à média de $1-2 \mathrm{~m} / \mathrm{s}$ durante o dia.

\section{B) Desenvolvimento do modelo de obtenção de densidade de área foliar}

A atenuação da radiação solar em meio vegetal é resultado primário da quantidade de elementos vegetais que interceptam os raios solares em seu percurso desde o topo até uma determinada altura no interior da vegetação. A orientação e a distribuição espacial desses elementos sâo outros fatores intervenientes que admitem e determinam configurações especiais da arquitetura da cobertura, responsáveis pelo grau de 
complexidade encontrado no estabelecimento e na obtenção das soluções de equações que governam o regime de radiação em cada tipo de cobertura. Em vegetação densa, algumas hipóteses sobre a variação desses fatores (distribuição espacial e orientação aleatória dos elementos vegetais são os exemplos mais comuns) simplificam o aparato matemático necessário. Nesses casos, a quantificação de área foliar existente em cada zona da vegetação, obtida de forma indireta a partir de medidas de radiação, torna-se factível e tem como ponto de partida o estabelecimento de uma relação (modelos físicos ou matemáticos) entre os fluxos de radiação solar e a área foliar acumulada (variação vertical preponderante), arranjados em equações do tipo,

$$
\alpha_{\mathrm{t}}=\alpha_{\mathrm{t}}(\mathrm{A})=\mathrm{F}_{\mathrm{t}}(\mathrm{A}) / \mathrm{F}_{\text {global }}(\mathrm{A}=0)
$$

Com a subsequente preparação do procedimento de inversão (expresso usualmente na forma de um algoritmo computacional) a função inversa que permite a estimativa de área foliar acumulada para cada ponto de interesse de uma mesma vertical é dada formalmente por,

$$
A=A\left(\alpha_{t}\right)
$$

sendo,

$$
A(z)=\int_{z}^{h} a(z) d z
$$

F fluxo de radiação (W.m $\mathrm{m}^{-2}$ )

A área foliar acumulada (adimensional)

a densidade de área foliar $\left(\mathrm{m}^{2} \cdot \mathrm{m}^{-3}\right)$

$\mathrm{z} \quad$ altura $(\mathrm{m})$

h altura da vegetação $(\mathrm{m})$

$\alpha$ fluxo relativo de radiação, que é dado pela razão entre o fluxo medido em uma altura $\mathrm{z}$ da vegetação e o fluxo no topo.

A associação entre fluxos relativos e área foliar acumulada, as definições particulares dos fluxos de radiação direta (proporção $\beta_{\mathrm{s}}$ ), difusa (proporção $\beta_{d}$ ) e complementar no interior da vegetação, o algoritmo básico de inversão de modelo de radiação e os valores adotados para os diferentes parâmetros, seguem no presente estudo a metodologia proposta por Wandelli \& Marques Filho (1999), que de forma resumida incorpora as seguintes equações:

$$
\begin{aligned}
\alpha_{\mathrm{t}}(\mathrm{A})= & \beta_{\mathrm{s}} \alpha_{\mathrm{s}}(\mathrm{A})+\beta_{\mathrm{d}} \alpha_{\mathrm{d}}(\mathrm{A})+\sum_{\mathrm{k}=1}^{\mathrm{n}} \gamma_{\mathrm{k}}\left(\beta_{\mathrm{s}} \alpha_{\mathrm{sl}, \mathrm{k}}(\mathrm{A})+\beta_{\mathrm{d}} \alpha_{\mathrm{dl}, \mathrm{k}}(\mathrm{A})\right. \\
\begin{array}{l}
\text { direta } \\
\text { Radiação primária }
\end{array} & \begin{array}{c}
\text { (n zonas do espectro) } \\
\text { Radiaçâo complementar }
\end{array}
\end{aligned}
$$

onde,

$$
\begin{array}{ll}
\alpha_{s}(A)=F_{s}(A) / F_{s}(A=0) & \alpha_{d}(A)=F_{d}(A) / F_{d}(A=0) \\
\alpha_{s 1}(A)=F_{s 1}(A) / F_{s}(A=0) & \alpha_{d 1}(A)=F_{d 1}(A) / F_{d}(A=0)
\end{array}
$$


$\alpha_{\mathrm{s}}\left[(\mathrm{A}(\mathrm{z})]=\mathrm{e}^{-\mathrm{k}(\theta \mathrm{s}) \mathrm{A}(\mathrm{z})}\right.$

$\alpha_{\mathrm{d}}[\mathrm{A}(\mathrm{z})]=\int_{0}^{\pi / 2} \mathrm{e}^{-\mathrm{k}(\theta) \mathrm{A}(\mathrm{z})} \cos \theta \cdot \operatorname{sen} \theta \cdot \mathrm{d} \theta$

$\left.\mathrm{F}_{1}(\mathrm{~A})=\mathrm{F}_{\mathrm{p} 1}(\mathrm{~A})-\frac{\Phi\left[\mu, \mathrm{r}\left(\mathrm{A}_{\mathrm{o}}-\mathrm{A}\right)\right]}{\Phi\left[\mu, \mathrm{rA}_{\mathrm{o}}\right]} \mathrm{F}_{\mathrm{p} 1}(0)-\frac{\Phi[1, \mathrm{rA}]}{\Phi[\mu, \mathrm{rA}]_{\mathrm{p} 2}} \mathrm{~F}_{\mathrm{o}}\right)$

$\Phi[x, y]=x e^{y}-\left(x e^{y}\right)^{-1}$

F fluxo de radiação complementar descendente

$\mathrm{r}, \mu$ paràmetros dependentes dos coeficientes de reflexão e transmissão dos elementos vegetais,

$K(\theta)$ coeficiente de extinção de luz $[=1 /(2 \cos \theta)]$ para a orientação aleatória dos elementos vegetais.

$\mathrm{Na}$ equação 3 , a radiação complementar no interior da cobertura vegetal é representada pela última parcela sob a somatória, cujos termos estão afetados pelos coeficientes de partição $\gamma_{k}$, compreendendo duas zonas do espectro eletromagnético com valores equivalentes e iguais a 0,5 , que representam a radiação fotossinteticamente ativa (RFA) e a radiação infravermelha (RIV).

As funções $\mathrm{F}_{\mathrm{p} 1}$ e $\mathrm{F}_{\mathrm{p} 2}$ surgem dos procedimentos de integração dos sistemas de equações diferenciais e são especificas aos termos primários de radiação direta e difusa. As formas adotadas no presente estudo são apropriadas para vegetação densa com distribuição espacial aleatória dos elementos vegetais e foram desenvolvidas no estudo de Marques Filho (1992).

Esses elementos de modelagem física da radiação solar em meio ve- getal constituem a base de apoio para a inversão matemática expressa na equação 2 , com a associação final entre os fluxos relativos no interior da cobertura e as estimativas correspondentes de área foliar acumulada; o procedimento de inversão materializa-se na forma de um programa computacional desenvolvido em linguagem de programação estruturada Fortran 77, e do ponto de vista matemático é simplesmente um procedimento numérico iterativo de inversão.

\section{C) Medidas de radiação}

Usualmente, tem-se adotado a utilização de mastros ou torres de estrutura metálica para a fixação dos instrumentos de medida. Essa estrutura de suporte, entretanto, interage com 0 ambiente proporcionando indesejáveis alterações nas grandezas medidas 
cujos valores serão tanto mais significativos quanto maior for a influência determinada por esta estrutura. Além disso, a dificuldade de deslocamento desses sistemas para realização de medidas em várias verticais internamente à vegetação representa um fator proibitivo a esse tipo de amostragem. Pensando nisso é que procurou-se, nesse trabalho, desenvolver um sistema de suporte que se utilizasse mais dos próprios elementos naturais existentes no ambiente de modo a reduzir ao máximo aquelas indesejáveis influências e, ao mesmo tempo, que apresentasse facilidade de translocação para obtenção de medidas em múltiplos pontos da vegetação (variabilidade horizontal). Dessa forma, foi criado um novo tipo de sistema com o aproveitamento do suporte natural representado pelas grandes árvores no sentido de suspender estruturas leves, flexíveis e modulares (Fig. 1) , formando um reticulado vertical de sustentação por cabos de aço unindo seções paralelas (previamente montadas) servindo de suporte aos sensores, cujo nivelamento horizontal é realizado durante o erguimento do reticulado, sempre à

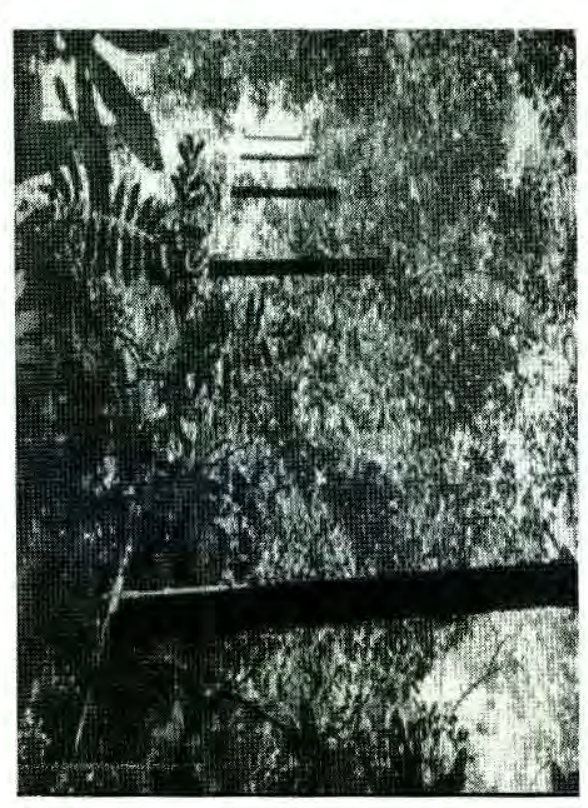

(a)

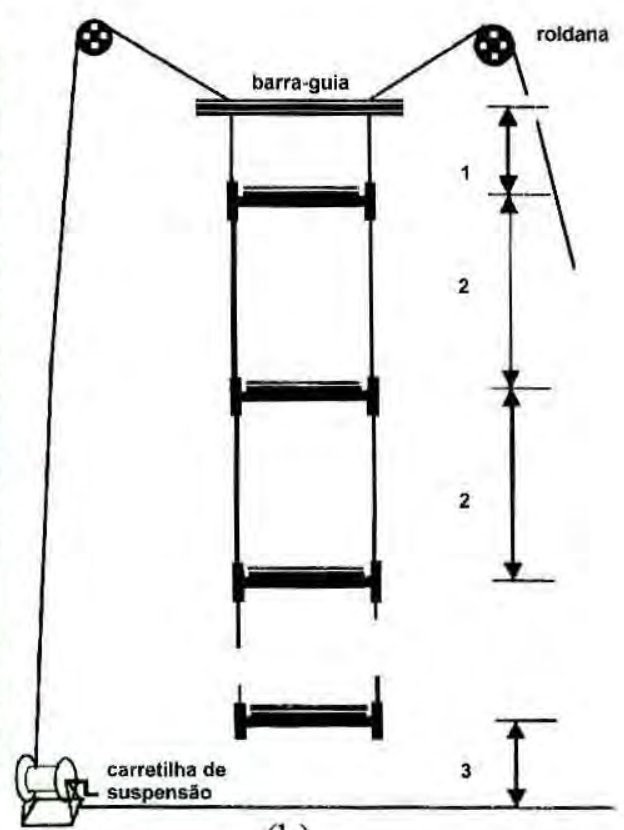

(b)

Figura 1. Fotografia do sistema de medidas (reticulado) instalado na Reserva Ducke (a); Esquema de representação do suporte reticulado utilizado para a distribuição dos sensores de medidas de radiação no interior do dossel: as distâncias $1(2 \mathrm{~m})$ e $2(5 \mathrm{~m})$ são fixas e a distância 3 variou em função da altura das árvores em que o reticulado foi suspenso (b). 
partir das duas seções mais próximas ao solo. No contexto desse novo sistema de medidas, a escolha dos pontos amostrais dá-se ao acaso, limitando-se apenas à existência dos suportes mais adequados representados pelas grandes árvores.

Os sensores foram fixados e nivelados nas diferentes seções (enquanto cada seção era suspensa) e os sinais foram medidos por um sistema automático de aquisição de dados (datalogger 21X, Campbell Scientific, Inc, England) disposto junto ao solo. A ascensão do conjunto (reticulado, sensores e cabos) foi feita por um sistema simples, constituído por dois dispositivos mecânicos (tipo carretilhas) fixos no solo, duas roldanas fixas na parte mais alta das árvores e cordas passando pelas roldanas e ligando as carretilhas ao reticulado de sustentação dos sensores. Os sensores utilizados foram os solarimetros tubulares Delta-T Devices, com superficie retangular de $2 \mathrm{~cm} \mathrm{X} 100 \mathrm{~cm}$ e sensibilidade à radiação de comprimentos de onda entre 0,3 e $3 \mu \mathrm{m}$ (intervalo característico do espectro solar), cujas medidas representaram integrações realizadas a intervalos de 20 minutos, no período diurno entre 6 e 18 horas.

Externamente à vegetação, as medidas de radiação solar global não apresentam, via de regra, influências determinadas pelo suporte dos seus sensores desde que estes possuam horizonte livre acima da sua superfície sensível. Assim, estas medidas foram obtidas de sensores dispostos no topo de uma torre micrometeorológica com cerca de $45 \mathrm{~m}$ de altura, presente na área. Os sensores utilizados para tanto foram um solarimetro tubular Delta $T$ e um piranômetro (medida auxiliar de controle) do tipo CM-5 Kipp \& Zonen (Delft, Netherlands) com intervalo de medidas entre 0,3 e $3 \mu \mathrm{m}$ e registros por integração a 20 minutos de intervalo, no período diurno entre 6 e 18 horas.

O período de medidas de radiação na floresta da Reserva Ducke estendeu-se de 29 de outubro a 11 de dezembro de 1998. O conjunto total de medidas representa a distribuição de radiação solar acima e no interior da vegetação em três verticais distintas nas proximidades da torre metálica existente no local. Os períodos específicos de funcionamento do dispositivo em cada vertical foram os seguintes: primeira vertical, de 29/10 a 06/11/98: segunda vertical, de 6 a 19/11/98: terceira vertical, de 19/11 a 11/12/98. Em cada vertical, o equipamento foi instalado (ascensão, posicionamento e nivelamento dos sensores, ligação dos cabos ao logger e preparação do programa são tarefas inerentes à instalação do sistema de medidas no campo) e mantido fixo durante todo o intervalo de dias correspondente àquela vertical.

A primeira vertical caracteriza a zona da vegetação situada abaixo dos 20 metros de altura (zona logo abaixo das copas das grandes árvores) e os tubos solarímetros foram dispostos 
nos níveis 0,$3 ; 3,6 ; 8,6 ; 13,6$; e 18,6 metros. As outras duas verticais cobrem a maior parte do espaço ocupado pela vegetação e tiveram pontos de medidas distribuídos nas alturas de 1,$7 ; 5,0 ; 10,0 ; 15,0 ; 20,0$; e 25,0 metros. Essas disposições particulares em cada vertical são decorrentes de fatores locais limitantes associados à arquitetura da vegetação (disposição espacial dos elementos todo o período de observação são agrupadas em quatro classes de radiação externa (I,II,III,IV), partindo de eventos em que predomina radiação difusa (céu encoberto, classes I e II) e alcançando aquelas ocasiões em que a radiação direta é dominante (céu limpo, classes III e IV), conforme representado na Figura 2.

Esses agrupamentos de dados viabilizaram a representação condensada do conjunto total de

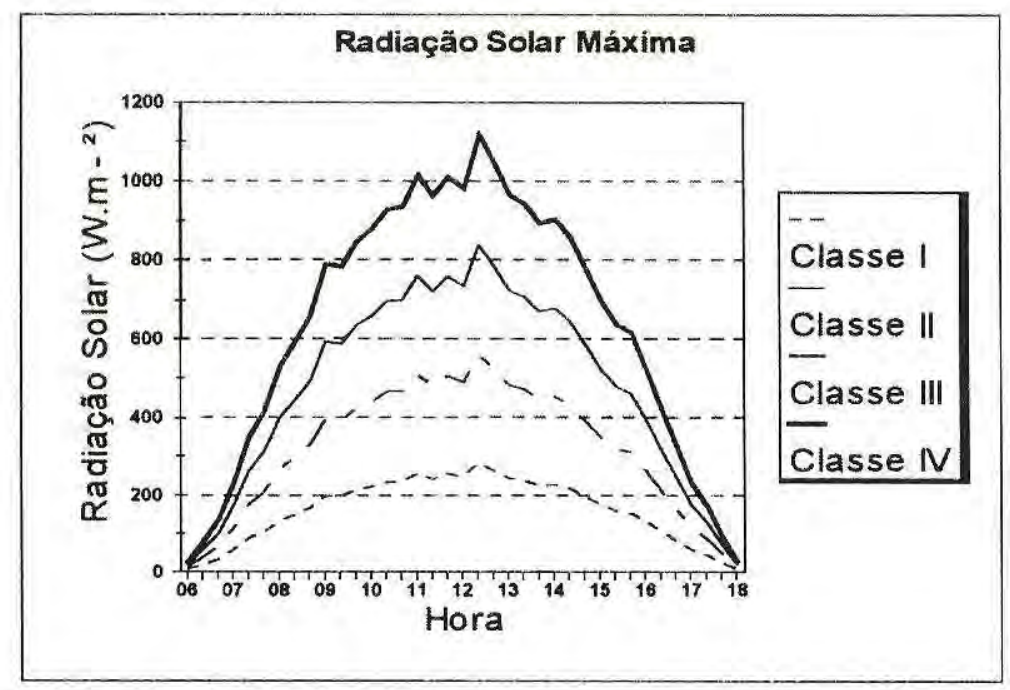

Figura 2. Classes de radiação externa: classe I - 0 a $25 \%$; classe II - 25 a $50 \%$; Classe III - 50 a $75 \%$; classe IV - 75 a $100 \%$.

vegetais) em confronto com as necessidades e restrições existentes para a colocação dos suportes superiores do dispositivo de medida (roldanas e cabos) na parte mais alta das grandes árvores.

\section{D) Processamento dos dados}

As medidas de radiação solar de informações acumuladas nas três verticais de observação (44 dias; 36 intervalos de 20 minutos; 7 pontos de cada vertical), resultando um total acima de 11000 valores médios medidos de radiação solar acima e no interior da cobertura. $\mathrm{O}$ tratamento desses dados gerou um número equivalente de estimativas de 
densidade de área foliar para os diferentes pontos no interior da floresta que precisavam ser organizadas para posterior interpretação.

Uma compactação ainda maior do conjunto de dados foi obtida seguindose o critério de separação por horários de observação, seguida do cálculo das médias representativas dos diferentes horários dentro de cada classe.

\section{RESULTADOS E DISCUSSÃO}

Com os procedimentos descritos acima, as variações temporais (curso diurno) dos fluxos relativos $a_{t}$ (e em correspondência os valores de área foliar acumulada, função $\mathrm{A}(\mathrm{z})$, estimados pelo algoritmo computacional associado a equação 2) são apresentadas em forma gráfica nas Figuras 3 a 8 . Em cada uma dessas figuras (em coordenadas polares e compostas por duas partes), os cursos diurnos médios dos fluxos relativos medidos em cada nivel são representados na parte superior; e os valores médios correspondentes estimados para a função $\mathrm{A}(\mathrm{z})$ de área foliar acumulada, na parte inferior. Observe-se a simetria axial adotada para a plotagem dos valores médios dos fluxos relativos e dos valores correspondentes calculados de área foliar. As Figuras 3 a 6 (classes I a IV, respectivamente) estão associadas a segunda vertical de observação; as Figuras 7 e 8 representam as condições de radiação difusa para as outras duas verticais. Em cada verti- cal, a convenção de traços das curvas é mantida para as quatro classes, e a associação entre as curvas e os niveis de medidas fica estabelecida na primeira figura de cada vertical pela regularidade das curvas da Classe I: na parte superior da figura, os niveis de medidas crescem a partir da curva mais interna; em conseqüência, na parte inferior, a correspondência ocorre no sentido inverso.

A comparação dos conjuntos de figuras permite identificar os traços marcantes presentes nos agrupamentos individuais (classes, verticais), que podem ser delineados nas seguintes proposições:

(i) A regularidade das curvas dos fluxos relativos (parte superior das Figs. 3 a 8 ) decresce com a presença da radiação direta. Em todas as verticais o comportamento temporal irregular dos valores médios observados dos fluxos relativos no interior da vegetação acentua-se nas condições de campo externo heterogêneo de radiação (radiação direta mais radiaçâo difusa, classes III e IV), em contraste às ocasiões próprias dos dias com cobertura de nuvens com baixo nivel de radiação, que determinam um campo externo de radiação mais homogêneo. Assim, as curvas diferenciam-se progressivamente em resposta à existência de uma direção dominante no campo externo de radiação, revelando de forma indireta, na flutuação dos fluxos relativos médios, a variabilidade intrínseca de área fo- 
liar e a sua distribuição irregular em diferentes zonas da vegetação. Uma observação seqüencial das Figuras 3 a 6 (segunda vertical, curva superior associada ao nível de 25 metros) revela um exemplo típico de tal variabilidade. Em condições de radiação difusa (Fig. 3) os fluxos de radiação são praticamente constantes ao longo do dia; em seguida (Fig. 4), alguns pequenos picos de radiação são perceptiveis para os horários de 9,10 e 17 horas; finalmente, com a predominância da radiação direta (Figuras 5 e 6) esses picos de radiação solar no interior da cobertura confirmam-se para os horários de $9 \mathrm{e}$ $17 \mathrm{~h}$. Esses eventos são possíveis em função do alinhamento da posição do Sol com espaços vazios ou de baixa densidade de área foliar da vegetação. As outras verticais também apresentam exemplos da variabilidade aqui tratada.

(ii) As curvas de área foliar acumulada (parte inferior das Figuras 3 a 8) têm flutuações correspondentes em cada horário e associadas aos fluxos relativos pelo modelo de inversão. Em termos relativos e considerando-se em cada curva as variações normalizadas $\left[\left(\mathrm{x}-\mathrm{x}_{\text {médio }}\right) /\right.$ $\left.\mathrm{x}_{\text {medio }}\right]$, essas flutuações são em geral menos acentuadas e de sentido oposto àquelas dos fluxos relativos, respeitando a natureza do fenômeno de interceptação de radiação em meio vegetal em que maiores fluxos de radiação estão associados a menores densidades de área foliar na zona considerada da vegetação.

(iii) Os efeitos da direcionalidade da radiação sobre os perfis medidos no interior da cobertura desaparecem nos casos em que a radiação externa medida não ultrapassa a $25 \%$ (classe I) da radiação máxima possível para o local e horário. Nesses casos o campo externo de radiação tende a ser marcadamente homogêneo e a descrição da interação radiação solar - vegetação é governada por um modelo puramente difuso.

(iv) A aproximação entre as curvas de fluxos relativos numa mesma vertical implica em baixa densidade de área foliar no espaço compreendido entre os dois pontos de medidas e deve ser esperada nos conjuntos de valores médios pertencentes às classes I e II. Exemplo típico deste fato é encontrado na Figura 7 (primeira vertical) em que as curvas representativas das alturas 13,6 e 18,6 metros têm cursos diurnos muito próximos e permitem inferir imediatamente a existência de um espaço vazio localizado ou uma baixa densidade de área foliar na zona de cinco metros em consideração. A consistência interna dos dados numa mesma vertical não é quebrada pela dissociação temporal e por uma eventual disparidade que possa aparecer entre as curvas correspondentes às classes III e IV. Esses comportamentos distintos entre classes não constituem elementos suficientes para se estabelecer uma contradição real e o conseqüente enfraquecimento da 


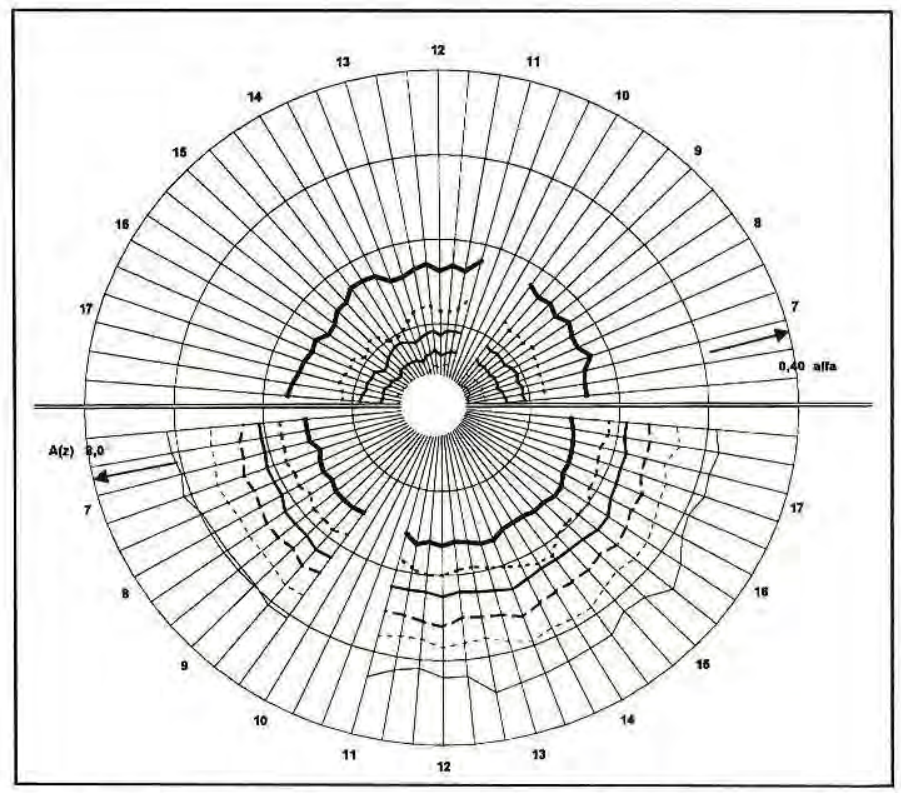

Figura 3. Curso diurno de Fluxos Relativos de radiação solar (parte superior) e valores correspondentes de Área Foliar Acumulada (parte inferior) em coordenadas polares. Segunda vertical, Classe I. Na parte superior da figura, os níveis de medidas crescem a partir da curva mais interna; em conseqüência, na parte inferior, a correspondência ocorre no sentido inverso.

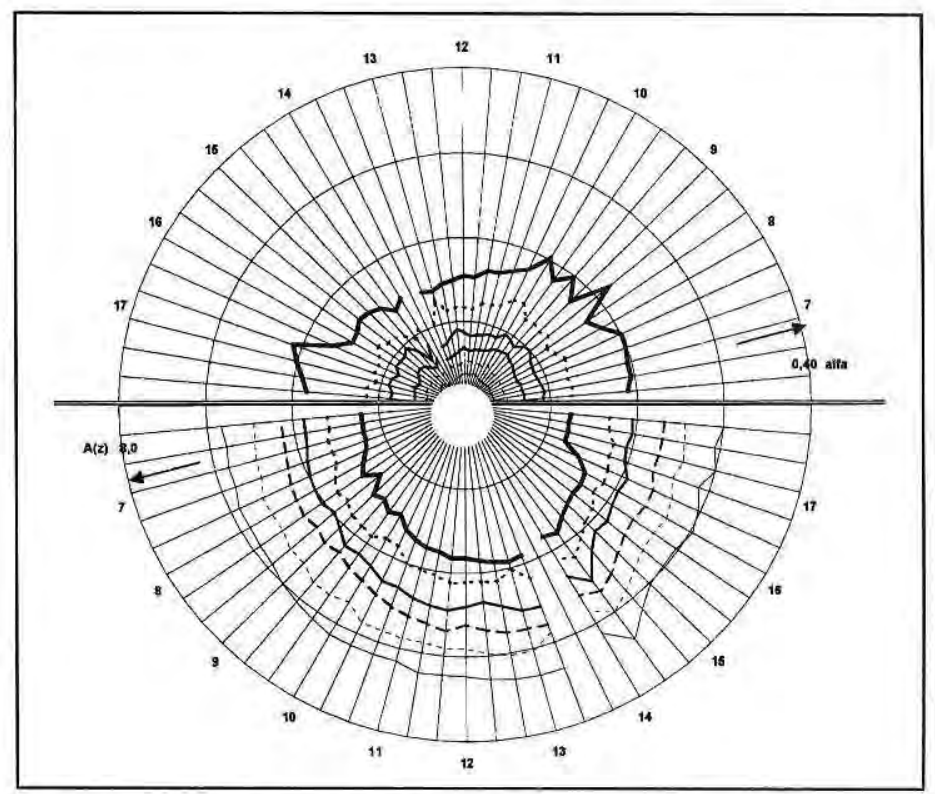

Figura 4. Curso diurno de Fluxos Relativos de radiação solar (parte superior) e valores correspondentes de Área Foliar Acumulada (parte inferior) em coordenadas polares. Segunda vertical, Classe II. Na parte superior da figura, os niveis de medidas crescem a partir da curva mais interna; em conseqüência, na parte inferior, a correspondência ocorre no sentido inverso. 


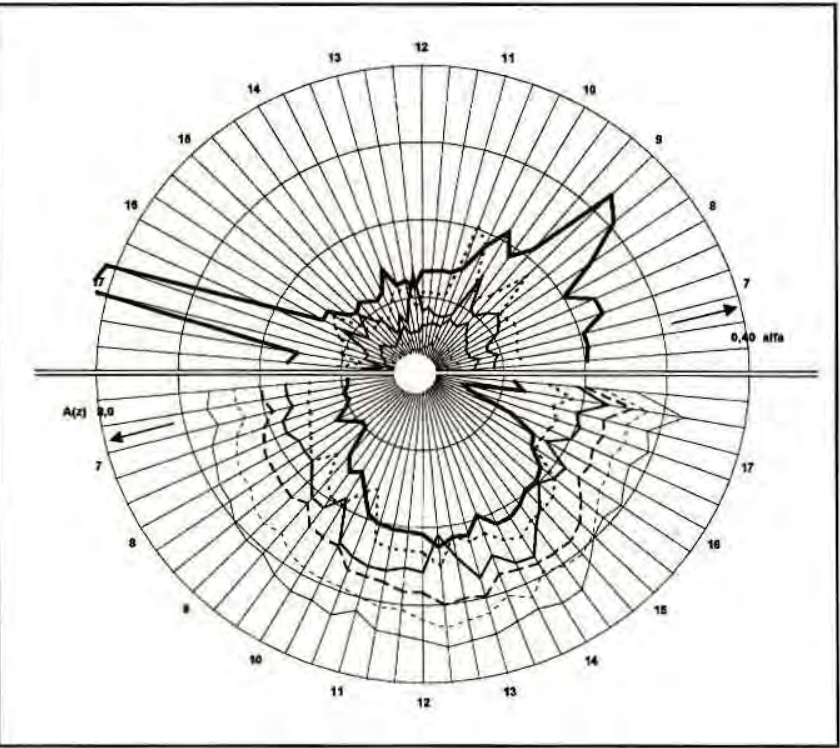

Figura 5. Curso diurno de Fluxos Relativos de radiação solar (parte superior) e valores correspondentes de Área Foliar Acumulada (parte inferior) em coordenadas polares. Segunda vertical, Classe III. Na parte superior da figura, os níveis de medidas crescem a partir da curva mais interna; em conseqüência, na parte inferior, a correspondência ocorre no sentido inverso.

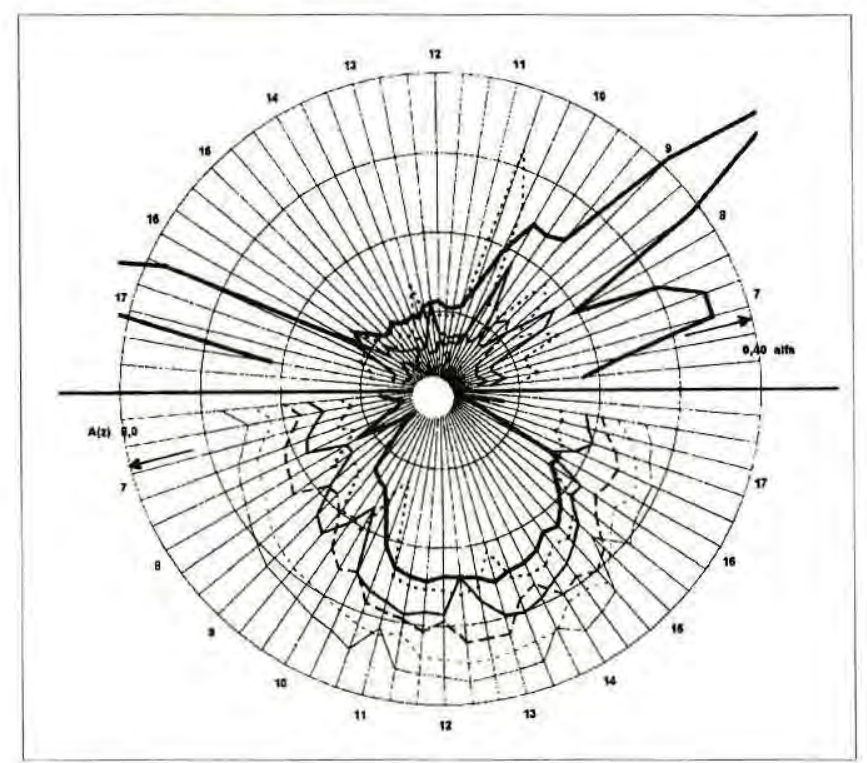

Figura 6. Curso diurno de Fluxos Relativos de radiação solar (parte superior) e valores correspondentes de Área Foliar Acumulada (parte inferior) em coordenadas polares. Segunda vertical, Classe IV. Na parte superior da figura, os niveis de medidas crescem a partir da curva mais interna; em conseqüência, na parte inferior, a correspondência ocorre no sentido inverso. 


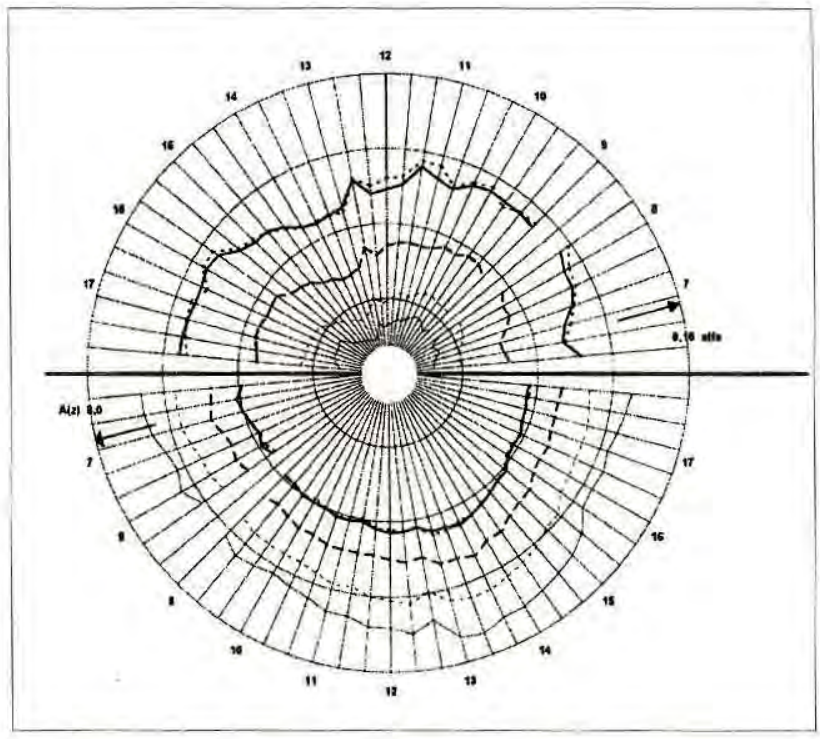

Figura 7. Curso diurno de Fluxos Relativos de radiação solar (parte superior) e valores correspondentes de Área Foliar Acumulada (parte inferior) em coordenadas polares. Primeira vertical, Classe I. Na parte superior da figura, os niveis de medidas crescem a partir da curva mais interna; em consequeencia, na parte inferior, a correspondência ocorre no sentido inverso. Esta vertical tem apenas cinco pontos de medidas

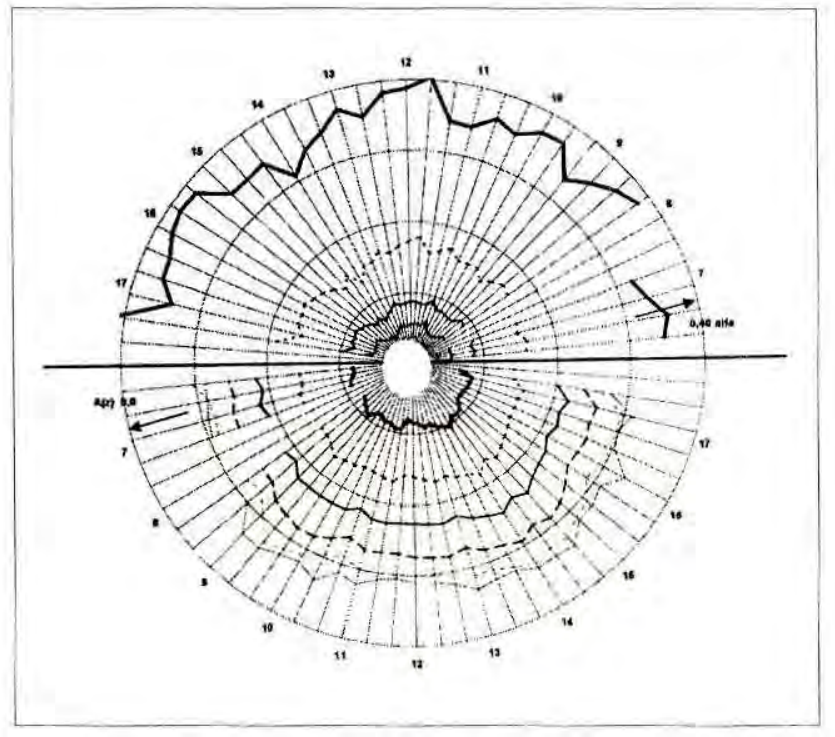

Figura 8. Curso diurno de Fluxos Relativos de radiação solar (parte superior) e valores correspondentes de Área Foliar Acumulada (parte inferior) em coordenadas polares. Terceira vertical, Classe I. Na parte superior da figura, os niveis de medidas crescem a partir da curva mais interna; em conseqüência, na parte inferior, a correspondência ocorre no sentido inverso. 
interpretação dada acima sobre a ocorrência de espaços vazios na cobertura, como poderá ser comprovado na seqüência da apresentação dos resultados.

Os valores médios de área foliar acumulada e desvios padrões correspondentes em diferentes níveis das três verticais de observação são apresentados nas Tabelas 1, 2 e 3, respectivamente. Essa operação de cálculo de médias é equivalente a integração dos efeitos individuais observados no curso diurno em cada classe, o que constitui uma forma de incorporar nas médias dos indices de área foliar distintas zonas da vegetação. A observação dos valores médios de cada classe para uma dada altura no interior da vegetação permite salientar um resultado de grande importância em estudos de inversão de modelos de radiação, a saber: a alta regularidade e a aproximação entre os valores médios das diferentes classes em um mesmo nivel de observação no interior da cobertura, um fato somente possível em função da integração de efeitos pelo cálculo de médias ao longo do curso diurno e explicável pela aproximação parcial de comportamento entre o modelo de radiação puramente difuso (campo de integração igual a $2 \pi$ esterorradianos) e o modelo mais geral com a ocorrência de radiação direta, que apresenta um ângulo de integração que se aproxima a $2 \pi$ radianos no intervalo diurno das 6 às 18 horas. .

A comparação dos perfis de área foliar acumulada, obtidos para as três verticais (IAF médio, terceira coluna das tabelas), fornece uma primeira indicação da variabilidade horizontal

Tabela 1. IAF calculado para cada classe de radiação. Valores entre parênteses representam o desvio padrão.

PRIMEIRA VERTICAL DE MEDIDAS

\begin{tabular}{|c|c|c|c|c|c|}
\hline \multirow{2}{*}{$\begin{array}{l}\text { Nivel de medida (m) no } \\
\text { interior da cobertura }\end{array}$} & \multicolumn{4}{|c|}{ IAF médio por classe de radiação } & \multirow{2}{*}{$\begin{array}{l}\text { IAF } \\
\text { Médio }\end{array}$} \\
\hline & 1 & II & 111 & IV & \\
\hline & $(0-25 \%)$ & $(25-50 \%)$ & $(50-75 \%)$ & $(75-100 \%)$ & \\
\hline$=0,3$ & $\begin{array}{c}6,57 \\
(0,37)\end{array}$ & $\begin{array}{c}6,16 \\
(0,33)\end{array}$ & $\begin{array}{c}6,37 \\
(0,62)\end{array}$ & $\begin{array}{c}6,35 \\
(0,89)\end{array}$ & 6,36 \\
\hline - 3,6 & $\begin{array}{c}5,73 \\
(0,31)\end{array}$ & $\begin{array}{c}5,50 \\
(0,33)\end{array}$ & $\begin{array}{c}5,77 \\
(0,66)\end{array}$ & $\begin{array}{c}5,98 \\
(0,81)\end{array}$ & 5,75 \\
\hline - 8,6 & $\begin{array}{c}4,83 \\
(0,24)\end{array}$ & $\begin{array}{c}4,68 \\
(0,32)\end{array}$ & $\begin{array}{c}4,90 \\
(0,64)\end{array}$ & $\begin{array}{c}5,22 \\
(0,83)\end{array}$ & 4,90 \\
\hline$-13,6$ & $\begin{array}{c}4,01 \\
(0,22)\end{array}$ & $\begin{array}{c}3,86 \\
(0,33)\end{array}$ & $\begin{array}{c}4,11 \\
(0,69)\end{array}$ & $\begin{array}{c}4,44 \\
(0,89)\end{array}$ & 4,10 \\
\hline$-18,6$ & $\begin{array}{l}4,00 \\
(0,23)\end{array}$ & $\begin{array}{c}3,81 \\
(0,32)\end{array}$ & $\begin{array}{l}4,09 \\
(0,73)\end{array}$ & $\begin{array}{l}4,27 \\
(0,96)\end{array}$ & 4,04 \\
\hline
\end{tabular}


Tabela 2. IAF calculado para cada classe de radiação. Valores entre parênteses representam o desvio padrão.

\section{SEGUNDA VERTICAL DE MEDIDAS}

IAF médio por classe de radiaçăo

II

111

IV

\begin{tabular}{cccccc}
\hline & $(0-25 \%)$ & $(25-50 \%)$ & $(50-75 \%)$ & $(75-100 \%)$ & \\
$-1,7$ & 6,33 & 6,06 & 6,22 & 6,33 & 6,23 \\
& $(0,47)$ & $(040)$ & $(0,54)$ & $(0,78)$ & \\
$-5,0$ & 5,48 & 5,39 & 5,62 & 5,84 & 5,59 \\
& $(0,25)$ & $(0,39)$ & $(0,59)$ & $(0,81)$ & \\
$-10,0$ & 4,90 & 4,80 & 4,98 & 4,97 & 4,91 \\
& $(0,26)$ & $(0,45)$ & $(0,64)$ & $(0,93)$ & \\
$-15,0$ & 4,35 & 4,25 & 4,34 & 4,48 & 4,36 \\
& $(0,19)$ & $(0,43)$ & $(0,75)$ & $(1,13)$ & \\
$-20,0$ & 3,86 & 3,71 & 3,80 & 3,79 & 3,79 \\
& $(0,25)$ & $(0,39)$ & $(0,73)$ & $(1,05)$ & \\
$-25,0$ & 3,15 & 3,02 & 3,17 & 3,01 & 3,09 \\
& $(0,18)$ & $(0,53)$ & $(0,99)$ & $(1,61)$ & \\
\hline
\end{tabular}

Tabela 3. IAF calculado para cada classe de radiação. Valores entre parênteses representam o desvio padrão.

TERCEIRA VERTICAL DE MEDIDAS

\begin{tabular}{|c|c|c|c|c|c|}
\hline \multirow{2}{*}{$\begin{array}{l}\text { - Nível de medida }(m) \text { no } \\
\text { interior da cobertura }\end{array}$} & \multicolumn{4}{|c|}{ IAF médio por classe de radiação } & \multirow{2}{*}{$\begin{array}{l}\text { IAF } \\
\text { Médio }\end{array}$} \\
\hline & 1 & 11 & 111 & IV & \\
\hline & $(0-25 \%)$ & $(25-50 \%)$ & $(50-75 \%)$ & $(75-100 \%)$ & \\
\hline$-1,7$ & $\begin{array}{c}6,19 \\
(0,54)\end{array}$ & $\begin{array}{c}5,79 \\
(0,33)\end{array}$ & $\begin{array}{c}5,76 \\
(0,61)\end{array}$ & $\begin{array}{c}5,63 \\
(1,02)\end{array}$ & 5,84 \\
\hline$-5,0$ & $\begin{array}{l}5,59 \\
(0,52)\end{array}$ & $\begin{array}{l}5,56 \\
(0,31)\end{array}$ & $\begin{array}{c}5,58 \\
(0,56)\end{array}$ & $\begin{array}{c}5,61 \\
(0,70)\end{array}$ & 5,67 \\
\hline - 10,0 & $\begin{array}{c}5,29 \\
(0,40)\end{array}$ & $\begin{array}{c}5,04 \\
(0,32)\end{array}$ & $\begin{array}{c}4,96 \\
(0,56)\end{array}$ & $\begin{array}{c}5,22 \\
(0,73)\end{array}$ & 5,13 \\
\hline$-15,0$ & $\begin{array}{c}4,42 \\
(0,33)\end{array}$ & $\begin{array}{c}4,19 \\
(0,41)\end{array}$ & $\begin{array}{c}4,05 \\
(0,69)\end{array}$ & $\begin{array}{c}3,95 \\
(1,29)\end{array}$ & 4,15 \\
\hline$-20,0$ & $\begin{array}{l}3,20 \\
(0,30)\end{array}$ & $\begin{array}{l}2,93 \\
(0,33)\end{array}$ & $\begin{array}{l}2,93 \\
(0,49)\end{array}$ & $\begin{array}{l}2,98 \\
(0,85)\end{array}$ & 3,01 \\
\hline$-25,0$ & $\begin{array}{r}1,71 \\
(0,27)\end{array}$ & $\begin{array}{c}1,53 \\
(0,23)\end{array}$ & $\begin{array}{c}1,44 \\
(0,46)\end{array}$ & $\begin{array}{c}1,34 \\
(0,83)\end{array}$ & 1,50 \\
\hline
\end{tabular}


de distribuição de área foliar da cobertura. A zona mais alta da vegetação onde concentram-se as copas das principais árvores apresenta as maiores discrepâncias entre os perfis (Tabelas 2 e 3 , valores associados aos níveis de 20 e 25 metros). Este fato é uma conseqüência natural da evolução, posicionamento relativo, e crescimento diferenciado das distintas espécies vegetais, que determinam a macro rugosidade normalmente observável acima de coberturas vegetais da Amazônia. Na zona intermediária e especialmente na zona inferior da vegetação próxima ao solo (zonas situadas abaixo dos 20 metros), os perfis nas três verticais apresentam sensível convergência.

Os perfis médios da função $\mathrm{A}(\mathrm{z})$ apresentados na última coluna das tabelas anteriores permitem a derivação numérica para vários pontos na vertical da densidade de área foliar a(z) a partir da expressão,

$a\left(z_{m}\right)=-d A\left(z_{m}\right) / d z=\left[A\left(z_{1}\right)-A\left(z_{2}\right)\right] /\left(z_{2}-z_{1}\right)$

sendo $\mathrm{z}_{\mathrm{m}} \circ$ ponto intermediário das alturas $\mathrm{z}_{1}$ e $\mathrm{z}_{2}$.

Nessa equação, a função $\mathrm{A}(\mathrm{z})$ deve ser representativa para a vegetação de um dado local e no presente estudo foi estabelecida como o perfil médio das três verticais com um índice de área foliar (IAF) total junto à superfície do solo dado pelo valor numérico de 6,36 (primeira vertical, nível 0,3 metros). Com esse procedimento, a distribuição vertical de área foliar foi determinada e aparece representada na Figura 9, em

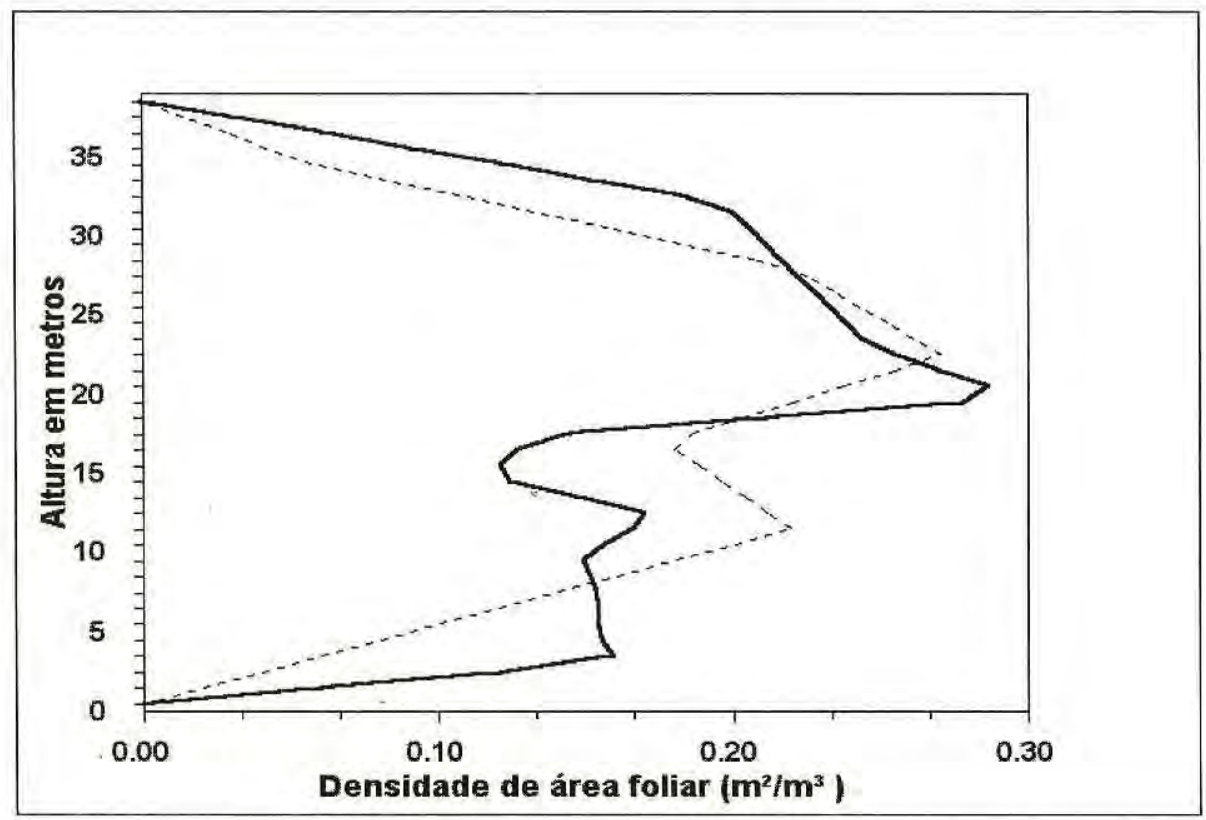

Figura 9. Densidade de área foliar, a(z); curva contínua corresponde ao estudo atual; curva tracejada corresponde ao estudo de Marques Filho (1997). 
confronto com uma curva similar de a(z) estabelecida para o mesmo local a partir de medidas de radiação fotossinteticamente ativa numa única vertical e tendo como suporte a torre metálica de $45 \mathrm{~m}$ de altura ali existente (Marques Filho, 1997). O detalhamento da distribuição vertical de $\mathrm{a}(\mathrm{z})$ no estudo atual é mais preciso em razão do maior número de pontos de observação especialmente na parte baixa da vegetação e da maior representatividade horizontal das medidas (três verticais). Na curva atual existem duas zonas distintas de concentração de área foliar: uma principal situada na parte alta da vegetação com concentração máxima de área foliar próxima de $0,3 \mathrm{~m}^{2} / \mathrm{m}^{3}$, localizada logo acima dos $20 \mathrm{~m}$ de altura; e uma secundária na parte baixa da vegetação, que apresenta densidade de área foliar praticamente constante da ordem de $0,15 \mathrm{~m}^{2} / \mathrm{m}^{3}$. Esses dois estratos são intercalados por uma zona de transição com baixa densidade de área foliar, cujo valor mínimo atinge $0,1 \mathrm{~m}^{2} / \mathrm{m}^{3}$ (localizada na altura de $15 \mathrm{~m})$.

\section{CONCLUSÃO}

A caracterização do regime de radiação solar em meio vegetal, combinada com a inversão de modelos físicos que representam a interceptação da radiação pela vegetação, produzem estimativas indiretas da distribuição espacial de área foliar, que são consistentes e representativas da cobertura na medida em que incorporam, no cálculo dos valores médios de cada ponto de uma dada vertical, a variabilidade natural de diferentes zonas da cobertura, condição satisfeita pela integração e pelo cálculo das médias ao longo do curso diurno em qualquer uma das classes de radiação adotada.

O sistema móvel de suporte dos sensores de radiação (reticulado modular) favorece a exposição dos sensores (redução de influências de superfícies estranhas à vegetação), permite amostragens detalhadas na zona inferior da cobertura (desde o solo até a altura de 20 metros) e em alguns pontos na parte alta da vegetação, e a sua concepção pode ser adaptada e estendida para qualquer medida micrometeorológica (temperatura, umidade, velocidade do vento, turbulência atmosférica) no ambiente interno de coberturas vegetais.

A função $a(z)$ de densidade de área foliar, estabelecida no presente estudo com dois estratos bem definidos, passa a representar no contexto atualizado de modelos radiativos a distribuição vertical média de área foliar da floresta da Reserva Ducke e associa a essa cobertura o valor de 6,4 como o indice de área foliar total (LAI).

\section{AGRADECIMENTOS}

Os autores agradecem aos Pesquisadores Elisa V. Wandelli da EMBRAPA e João Dantas de Oliveira do INPA pela discussão e sugestões apresentadas sobre o manuscrito; aos técnicos da Coordenação de Pesquisas 
em Geociências do INPA: Afonso Ligório da Mota, Hermes Braga Xavier, Jauapery Neves Pereira Júnior, Sérgio Magno Valério de Souza e Valdelice Pereira da Silva; pela ajuda na preparação e instalação dos sistemas de medidas; ao estagiário Alessandro Michiles pela ajuda na instalação e coleta dos dados. Este estudo se insere no Projeto de Pesquisa "Balanço de energia, vapor d'água e $\mathrm{CO}_{2}$ em área de floresta tropical na Amazônia Central", financiado sob a referência PPD 95/ 0966.

\section{Bibliografia citada}

Andrieu, B.; Baret, F. 1993. Indirect methods of estimating crop structure from optical measurements. In: Varlet-Grancher, C.; Bonhomme, R.; Sinoquet, H. (Eds.) 1993 Crop Structure and Light Microclimate. INRA, France. p. 285-322.

Honzák, M.; Lucas, R. M.; Amaral. I.; Curran, P. J.; Foody, G. M.; Amaral, S., 1996. Estimation of the leaf area index and total biomass of tropical regenerating forests: comparison of methodologies, In: Gash, J.H.C.; Nobre, C.A.; Roberts, J.M.; Victoria, R. L.(Eds.) 1996. Amazonian Deforestation and Climate. Institute of Hydrology, UK. p, 365-381.

McWilliam, A. L. C.; Roberts, J. M.; Cabral, O. M. R.; Leitão, M. V. B. R.; Costa, A. C. L.; Maitelli, G. T.; Zamparoni, C. A. G. P. 1993. Leaf area index and above ground biomass of terra firme rain forest and adjacent clearings in Amazonia. Functional Ecology, 7: 210-217.
Marques Filho, A.O. 1992. Modèles des transferts radiatifs à l'intérieur des couverts végétaux - les solutions analytiques. Acta Amazonica, 22(4):541565.

Marques Filho, A.O. 1997. Regime de radiação solar e características da vegetação Modelos de inversão. Acta Amazonica, 27(2):119-134.

Myneni, R.R.; Ross, J.; Asrar, G. 1989. A review on the theory of photon transport in plant canopies. Agricultural and Forest Meteorology, 45: 1-153.

Nilson, T. 1971. A theoretical analysis of the frequency of gaps in plant stands, Agricultural and Forest Meteorology, 8: 25-38.

Roberts, J. M.; Cabral, O. M. R.; Costa, J. P.; McWilliam, A. L. C.; Sá, T. D. A. 1996. Leaf area index, biomass and physiological responses in Amazonian forest and pasture. In: Gash, J.H.C.; Nobre, C.A.; Roberts, J.M and Victoria R. L. (Eds). Amazonian Deforestation and Climate. Institute of Hydrology, UK. p. 287-306.

Ross, J. 1981. The Radiation Regime and the Architecture of Plant Stands. Dr. W. Junk Publ., The Netherlands.

Wandelli, E. V.; Marques Filho, A. O. 1999. Medidas de radiação solar e indice de área foliar de coberturas vegetais. Acta Amazonica, 29(1): 57-78.

Welles, J. M.; Norman, J. M. 1991. Instrument for indirect measurement for canopy architecture. Agronomy Journal, 53:818-825. 